\title{
A Simple Method of Managing the Alveolar Antral Artery during Sinus Lift Surgery
}

\author{
David H. Yang1* ${ }^{1}$, Nathan V. Lee ${ }^{2}$ \\ ${ }^{1}$ Oral and Maxillofacial Surgery, British Columbia Cancer Agency, Faculty of Dentistry, University of British Columbia, \\ Vancouver, Canada \\ ${ }^{2}$ Oral Medicine and Oral Pathology, Faculty of Dentistry, University of British Columbia, Vancouver, Canada \\ Email: *david.yang@bccancer.bc.ca
}

How to cite this paper: Yang, D.H. and Lee, N.V. (2021) A Simple Method of Managing the Alveolar Antral Artery during Sinus Lift Surgery. International Journal of Otolaryngology and Head \& Neck Surgery, 10, 131-146.

https://doi.org/10.4236/ijohns.2021.103014

Received: March 8, 2021

Accepted: May 4, 2021

Published: May 7, 2021

Copyright $\odot 2021$ by author(s) and Scientific Research Publishing Inc. This work is licensed under the Creative Commons Attribution International License (CC BY 4.0).

http://creativecommons.org/licenses/by/4.0/

\begin{abstract}
The alveolar antral artery resides lateral to the maxillary sinus and can lead to complications in sinus lift surgery. Traditional approaches that decrease intraoperative bleeding into the surgical field include vessel preservation using multiple bony windows or neutralizing the vessel at the surgical site. Unfortunately, these methods are technique sensitive, time intensive, and may lead to hemosinus and graft loss. The variable distance from the crest of the alveolar ridge and vessel diameter further complicates pre-operative planning. This paper discusses the anatomical features of the alveolar antral artery, techniques for clinical assessment, and current management strategies. We then describe a novel protocol to manage the alveolar antral artery in sinus lift procedures via tamponade of the vessel at a proximal site. This method is faster than those described in the literature, does not require any additional equipment or expertise, and aims to improve long-term graft predictability by decreasing the risk of sinus membrane perforation. The alveolar antral artery is an under-reported source of surgical complications and warrants further research.
\end{abstract}

\section{Keywords}

Alveolar Antral Artery, Maxillary Sinus Augmentation, Dental Implants, Sinus Lift, Maxillary Sinus Membrane, Bone Graft

\section{Introduction}

A lateral window sinus augmentation is a workhorse of an implant surgery practice. Decades after its first description by Tatum, augmentation of the deficient 
sinus floor to facilitate endosteal implant placement has become routine [1]. Paradigm changes in the last decade or so have given us alternatives; crestal approach sinus lifts and graftless solutions with tilted and zygomatic implants all bypass the conventional sinus lift. But crestal approaches have poor predictability in the severely atrophic maxilla, and graftless solutions are currently only indicated for full-arch therapy [2] [3]. Thus, there remains an indication for this classic sinus lift via a lateral window in many cases.

The alveolar antral artery (AAA) is a commonly encountered vessel in the surgical field of a sinus lift. This bleed is a "gateway complication", of which disruption may cause further downstream complications that impact treatment prognosis and outcome. Hemorrhage from the AAA can then lead to longer surgical time, poor visibility, membrane perforation, graft migration from "wash-out", hemosinus causing sinus dysfunction and infection, and hematoma formation leading to higher infection risk and graft loss [4]. The most common intra-operative complication, perforation of the sinus membrane, has been reported in $10 \%$ of cases and $1 \%$ of sinus augmentation procedures had to be aborted. Infection and maxillary sinusitis are the most common post-operative complications, occurring in $3 \%$ of cases and contributing to partial or complete graft loss in under $1 \%$ of cases [5]. Literature regarding the exact incidence of AAA-related complications is under-reported. The vessel is associated with higher detection rates in males due to larger diameters, as well as in narrower maxillary sinuses $<14 \mathrm{~mm}$ in width, two groups that may be at higher risk for intraoperative laceration [4]. Current literature suggests two main strategies during a sinus lift:

1) Maintain the vessel via a double-window approach or piezoelectric approach [6].

2) Neutralize the lacerated vessel at the site of the osteotomy; either prophylactically or reactively when encountered.

Normally positioned lateral wall osteotomies would interfere with the AAA in $10 \%-30 \%$ of cases [7] [8] [9]. Bleeding from this vessel can persist in the immediate post-operative period or be delayed up to seven hours after surgery due to the use of vasoconstrictors [10]. These vascular complications may compromise surgical outcomes [11]. While some papers have described the AAA as a small artery whose laceration does not impact the outcome of the procedure, [12] others have found the risk of significant bleeding to be $10 \%-20 \%$ [8] [13] [14]. There can be great variation in the path, diameter, and position of the AAA. Given the vessel's relationship with the feeding maxillary artery, encountering this artery intra-operatively could lead to brisk bleeding [7]. In fact, these same papers that have argued against considering the AAA also state that studies that investigate characteristics of the AAA that impact its effect on sinus augmentation success rate are lacking and have recommended further research [12].

We present an alternative method of managing this artery that may be simpler and more predictable without adding any additional intra-operative time. 


\section{Anatomy}

The AAA is the anastomosis of the Posterior Superior Alveolar Artery (PSAA) and the Infraorbital Artery (IOA). It has a curvilinear path, roughly paralleling the sinus floor, with the lowest position in the second premolar and first molar area, which are the most common sites needing augmentation for implant therapy (Figure 1(a) and Figure 1(b)). The vessel is at its largest diameter at the first molar area, [15] which also happens to be the most common site to be augmented via a lateral window. The average diameter has been shown to be 1.5 $\mathrm{mm}$ [16]. Diameters $<0.5 \mathrm{~mm}$ tend to pose no significant bleeding to interfere with surgery [12]. However, Testori et al. [17] noted that a small caliber vessel radiographically may correspond to a much larger caliber vessel clinically.

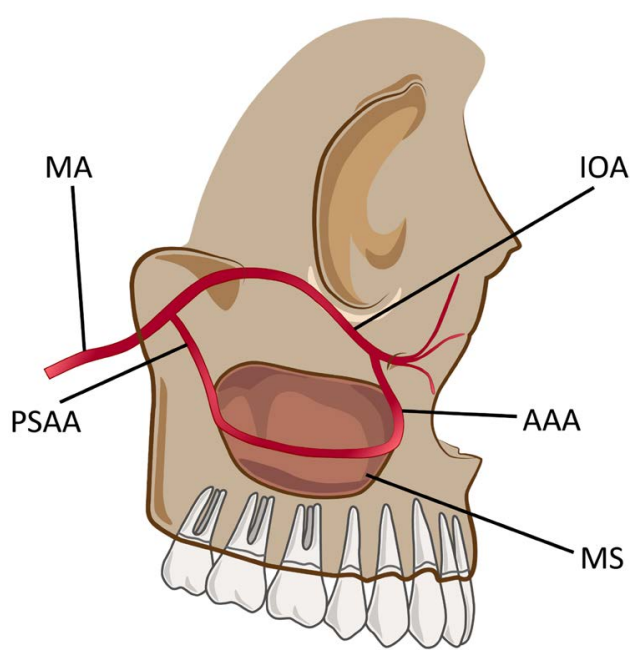

(a)

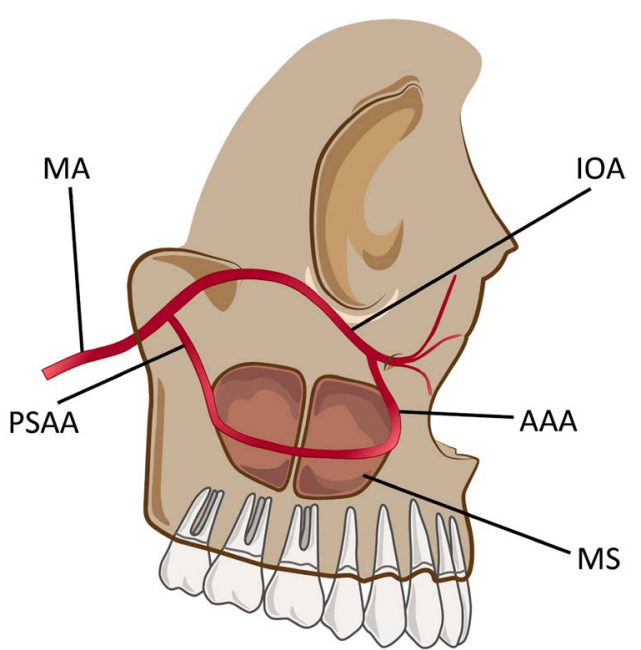

(b)

Figure 1. Anatomy of the alveolar antral artery passing laterally to a single maxillary sinus (a) and septated sinus (b). It forms an anastomosis with the infraorbital artery anteriorly and the posterior superior alveolar artery posteriorly. AAA, alveolar antral artery; IOA, infraorbital artery; MA, maxillary artery; MS, maxillary sinus; PSAA, posterior superior alveolar artery. 
Larger caliber vessels can lead to significant bleeding when they occur in the path of planned osteotomy. Ella et al. [13] estimated this risk with respect to the AAA to be $>10 \%$ while Chan \& Wang [14][14] as well as Elian et al. [8] projected the risk to be $20 \%$. Severe bleeding from a transcrestal sinus lift, presumably from a lacerated AAA by an osteotomy path close to the lateral sinus wall has also been reported [18].

The distance of the AAA from the alveolar ridge varies greatly and may depend on whether dentate or edentulous patients are selected, as ridge resorption in edentulism or associated with age-related changes will reduce this distance [19] [20]. Shortest distance between the alveolar crest and AAA occurs at the first and second molar regions [21]. Solar et al. [22] found a range of 15 - $25 \mathrm{~mm}$ of alveolar bone height in a mixed dentate/edentulous population (Figure 2(a) and Figure 2(b)).

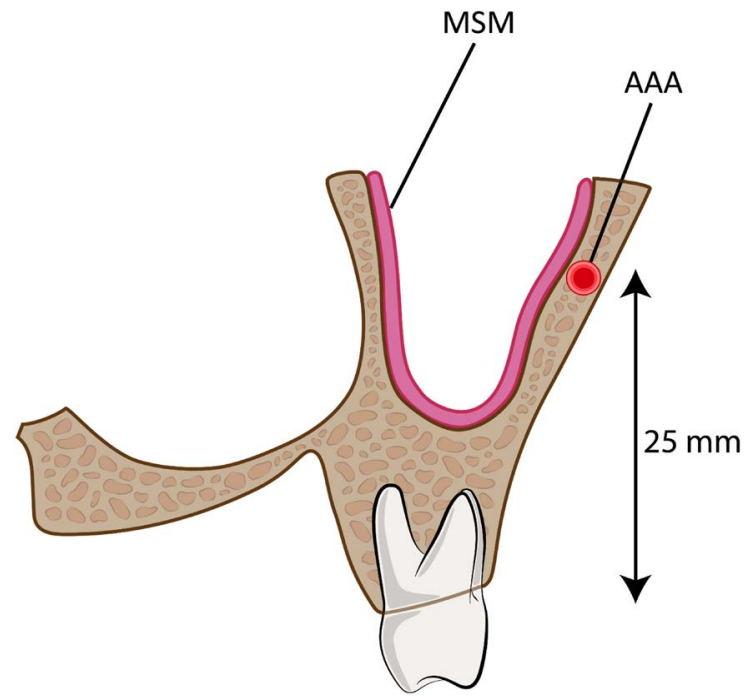

(a)

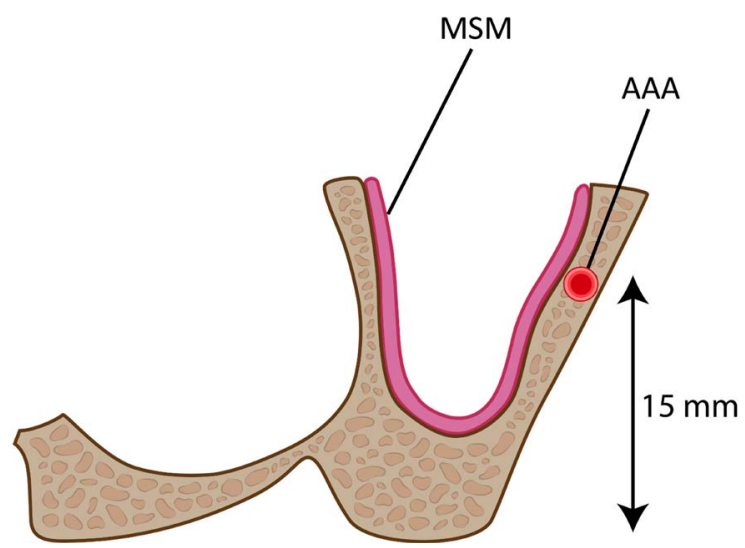

(b)

Figure 2. Frontal plane schematic of the alveolar antral artery and maxillary sinus. On average, the distance from vessel to alveolar crest is $25 \mathrm{~mm}$ in a dentulous maxilla (a) and $15 \mathrm{~mm}$ in an edentulous maxilla (b). Ridge resorption is one of the causes of bone height variation. AAA, alveolar antral artery; MSM, maxillary sinus membrane. 
A more constant parameter that is less impacted by ridge resorption would be the vertical distance of the AAA from the sinus floor, which has been found to have a mean of $7.66 \mathrm{~mm}$ in a cone beam computed tomography (CBCT) series of 240 sinuses [23]. Similarly, Park et al. [24] found this height to be between $7.71-8.01 \mathrm{~mm}$, and proposed that a minimal window height of $8 \mathrm{~mm}$ be used to allow visualization as well as simple instrumentation and graft placement. This means if one places the osteotomy at the lowest possible position at the base of the sinus, it would be possible to avoid the vessel altogether in some cases (Figure 3(a), Figure 3(b), and Figure 3(c)). If the osteotomy was placed slightly higher up the sinus wall, then the AAA being in the path of the osteotomy becomes almost a certainty (Figure $3(\mathrm{~d})$ ).

The further the AAA is from the crest, the larger its diameter [25]. While some authors have dictated that vessels $>15 \mathrm{~mm}$ from the crest can be safe as this

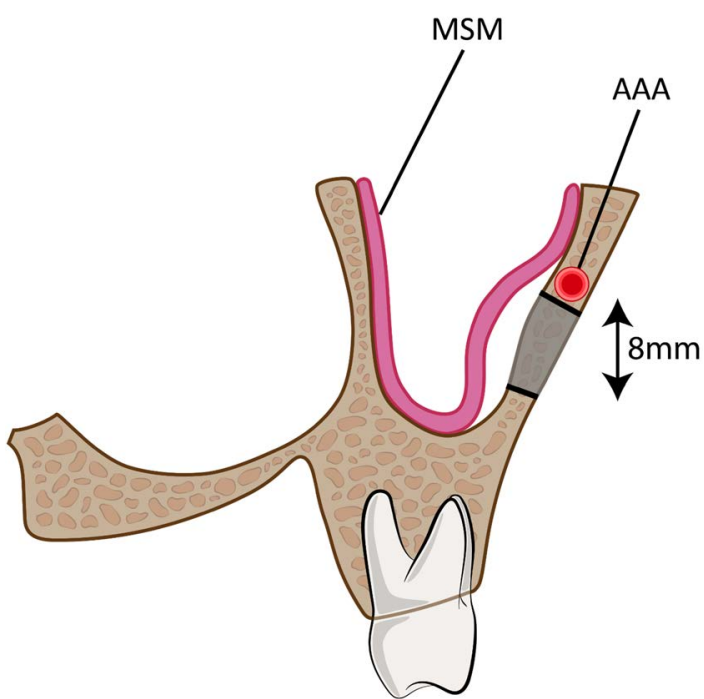

(a)

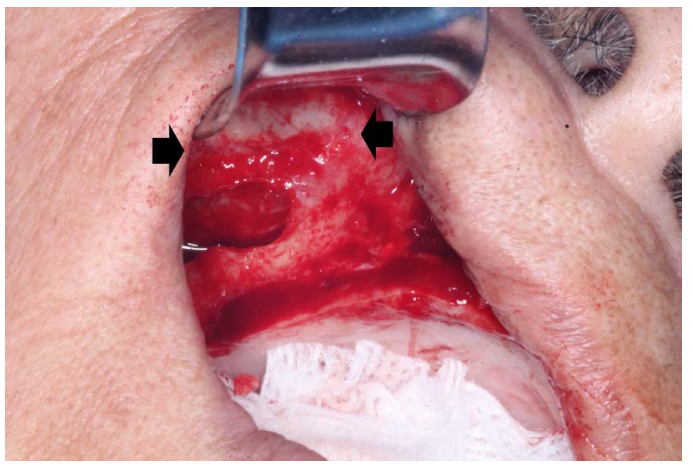

(c)

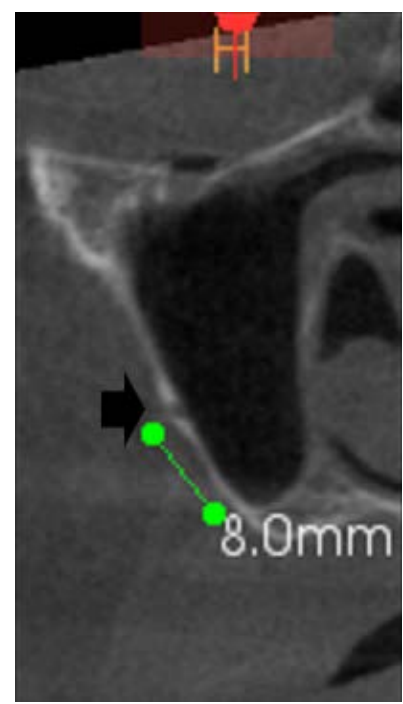

(b)

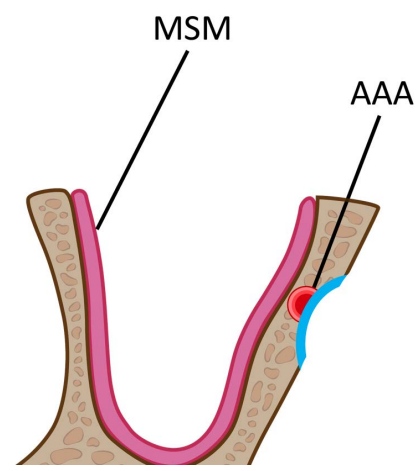

(d)

Figure 3. To preserve the alveolar antral artery, a minimum $8 \mathrm{~mm}$ bony window height inferior to the vessel is recommended (a). Cone beam computed tomography imaging (b) shows an appropriate distance between the alveolar antral artery (black arrow) and the sinus floor. The osteotomy was designed below the intact alveolar antral artery to avoid it all together (c). Our proposed osteotomy protocol (d) allows sacrifice of the vessel if there is not enough height. AAA, alveolar antral artery; MSM, maxillary sinus membrane. 
represents the maximum graft height required, [12] [25] [26] [27] [28] at times a higher bone level is needed. Various forms of overdenture prosthetic designs demand a minimum of 13 - $15 \mathrm{~mm}$ of implant-to-occlusal clearance. When this height does not exist naturally, alveolectomy to create clearance would need an accompanying higher graft level to compensate. Posterior maxillae which have migrated inferiorly from longstanding edentulism and sinus pneumatization would compound this problem (Figure 4).

\section{Detection}

CBCT evaluation is the best way to screen for the presence of this vessel. On coronal cuts, the AAA presents as a discrete radiolucency within the bony sinus wall. This finding is only detectable in approximately $50 \%$ of pre-operative CT scans, [8] [12] [29] [30] but it would be incorrect to assume the other $50 \%$ as an "absent vessel". It has been shown in multiple cadaver studies that this anastomosis is present $100 \%$ of the time [31]. The undetectability is attributed to:

1) extrabony vessel occurring lateral to the sinus wall.

2) intrabony vessel of too small a caliber be detectable on CT [30] [32].

3 ) intrasinus vessel occurring within the sinus, between the sinus wall and membrane (Figure 5).

The extrabony vessel may be retracted with the flap. If lacerated during flap access, it is easily managed by a variety of techniques without risk of damaging the sinus membrane, including electrocautery, ligation, and gauze tamponade.

Disruption of intrabony vessels, when of significant caliber, immediately causes a loss of visibility directly into the surgical field. This disruption tends to occur during the initial elevation of the membrane when visibility is most critical. The intrasinus vessel is also difficult to manage as it is intimately adhered to the membrane but does not always readily reflect upwards with the membrane owing to its intrabony origins proximally and distally.

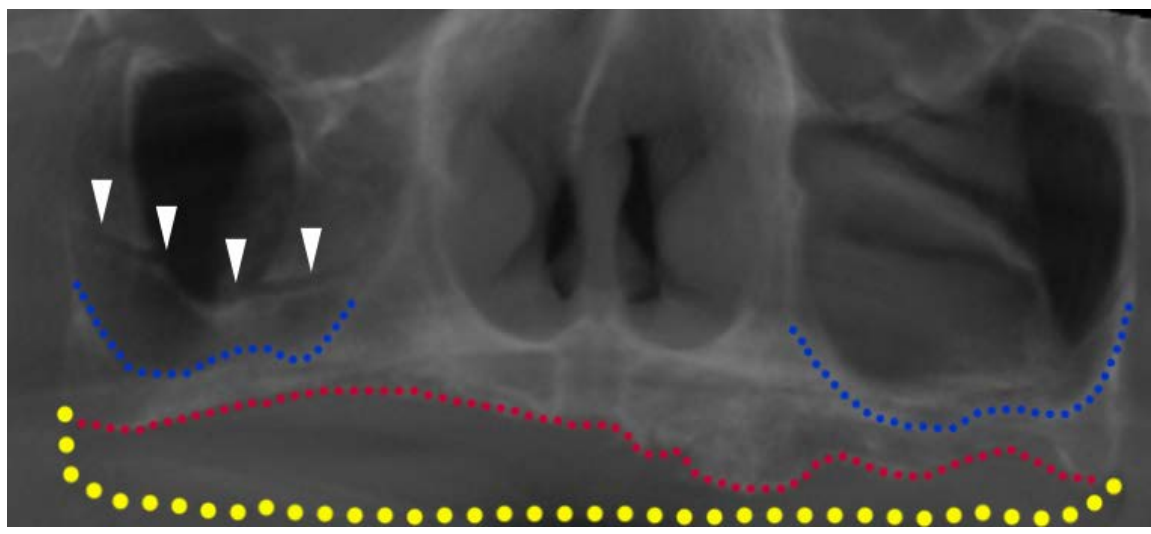

Figure 4. The three dotted lines from upper to lower, denote the sinus floor (blue), existing resorbed ridge height (red), and pre-existing ridge height (yellow) in a long-term edentulous patient. This resorption requires the clinician to augment the sinus floor by 10 - $12 \mathrm{~mm}$, which increases the likeliness of encountering the alveolar antral artery (white arrowheads) intraoperatively. 


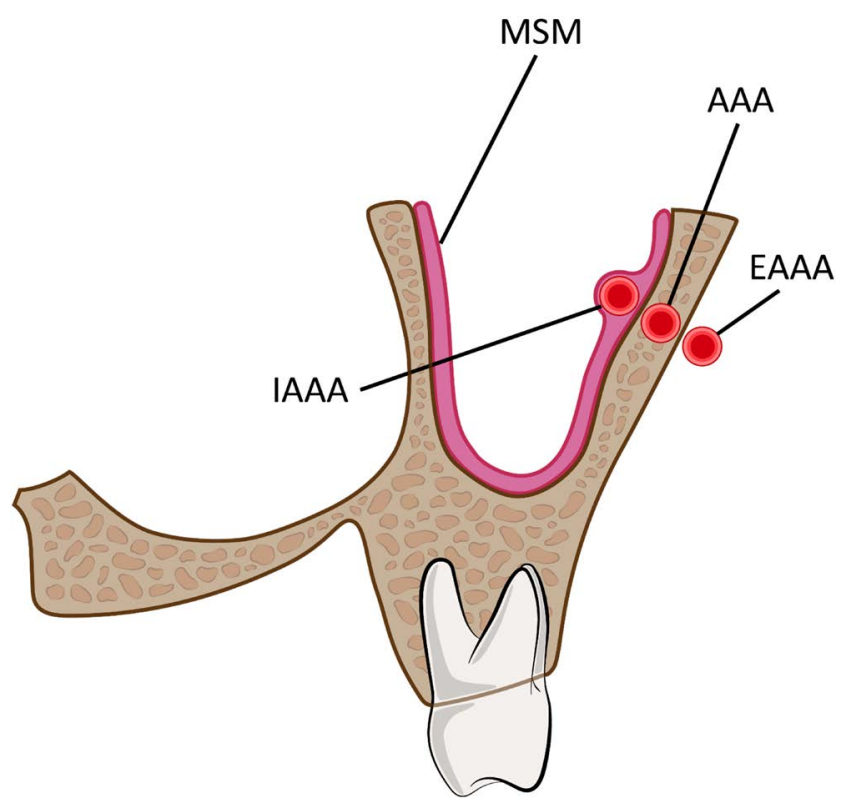

Figure 5. Difficulty in detection of the alveolar antral artery by cone beam computed tomography can be attributed to the variation in its course. AAA, alveolar antral artery (intrabony); EAAA, extrabony alveolar antral artery; IAAA, intrasinus alveolar antral artery; MSM, maxillary sinus membrane.

\section{Current Management Strategies}

1) Tamponade

Hemostatic agents have been suggested [33]. They work by enhancing the coagulation cascade on a molecular level, acting as a mechanical obstruction to promote clot formation macroscopically, or both. In an arterial flow situation, the coagulation cascade plays no role as any applied agent is washed out immediately. A macroscopic disruption of the flow is first needed before coagulation can occur.

Flanagan described packing graft material directly into the bony canal to achieve tamponade [34]. Lee recommend gauze sponge saturated with topical thrombin for prolonged bleed lasting 45 minutes, when other approaches had failed [7]. However, a rigid bony canal that allows material compression does not always exist. There may be a thin or absent wall, often on the inner table, such that material blindly packed into the canal opening simply extrudes via the wall defect into the sinus and possibly damages the sinus membrane.

Bone wax requires either a roughened, undercut area to provide mechanical retention or a broad, dry surface to which to adhere, both of which do not exist in this scenario [35]. The main concern with crushing bone into the area would be membrane damage [16].

Any strategy requiring tamponade would automatically extend surgical time while waiting for clot formation, as whatever material used would block surgical vision and access. Even after a clot is formed, further instrumentation may retrigger bleeding, requiring the procedure to be aborted, or completed with sig- 
nificant increase in surgical time.

If the membrane is torn at any point of this bleeder management, blood can pool inside the sinus proper of the supine patient, leading to post-operative hemosinus. This can impair osteomeatal complex patency and increase risks of infection, all which ultimately lead to graft failure.

All the strategies described above assume an intrabony vessel. Specifically, they presume the presence of a bony separation, albeit thin, between the vessel and the sinus membrane. However, in $13 \%-52.8 \%$ of cases, the vessel is intrasinus and directly adherent to the sinus membrane, making vessel management without perforation difficult or impossible [36].

2) Electrocautery, chemocautery, and ligation

With cautery, especially when monopolar, there is no control of the exact area of cauterization. The capillary network of the membrane vasculature can be damaged beyond the visualized area of tissue desiccation. Thus, even when no visible membrane tear occurs, subsequent necrosis of an area of the Schneiderian membrane directly over the graft may present delayed post-operative issues. Chemocautery with silver nitrate would carry similar concerns of local destruction and, in the authors' experience, is ineffective in arterial bleeds. Testori et al. have described ligation of a $3 \mathrm{~mm}$ diameter vessel in the path of the osteotomy after its isolation [17].

3) Delayed management

Some surgeons advocate lifting the nearby membrane first while ignoring the bleeder, such that hemostatic strategies can be done safely later with the membrane retracted well away. The bleed can be temporary neutralized with a well-placed suction directly on the bony canal, and the membrane is lifted with good visibility. However, if the membrane tears during elevation, the potential for a hemosinus to pool via the torn membrane opening would present yet again.

4) Preservation

Piezoelectric devices may be used to cut the bony window without violating the membrane or vessel [37]. This may allow intra-antral vessels to reflect upwards with the sinus membrane. Invariably, the AAA is tethered to bone on both ends since the IOA and PSAA arteries are extrabony structures when their anastomoses begin. Therefore, the vessel may prevent proper membrane elevation and may tear at the time of graft packing. This approach is technique sensitive and can be time consuming. Palatal approaches have been described but are limited to select cases [38].

Maridati et al. [39] describe a double-window technique (Figure 6(a)), where osteotomies are made above and below the vessel leaving a thin bridge of bone holding the intact vessel. This is a difficult approach and is not always possible to reproduce as some vessels would be positioned in a way that makes the inferior window very narrow and difficult to instrument. This is further complicated by bifid vessels, or septated sinuses (Figure 6(b)). Presence of at least one septum is found in $44.4 \%$ - 58\% of patients [30] [40] [41] [42]. Septum height has a mean of $8.22 \mathrm{~mm}$ but ranges from 5.6 - $20.6 \mathrm{~mm}$. [43] 


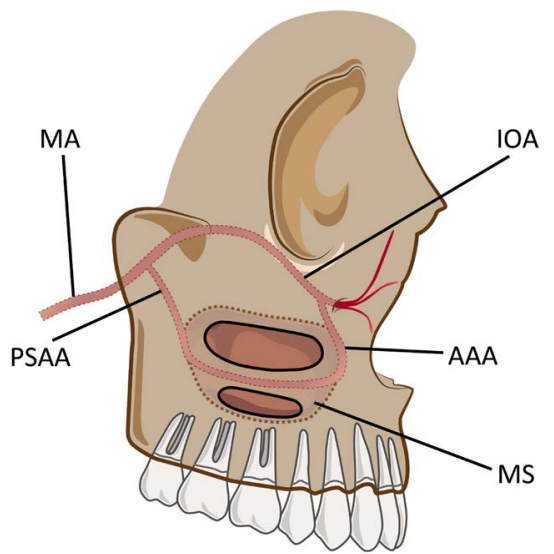

(a)

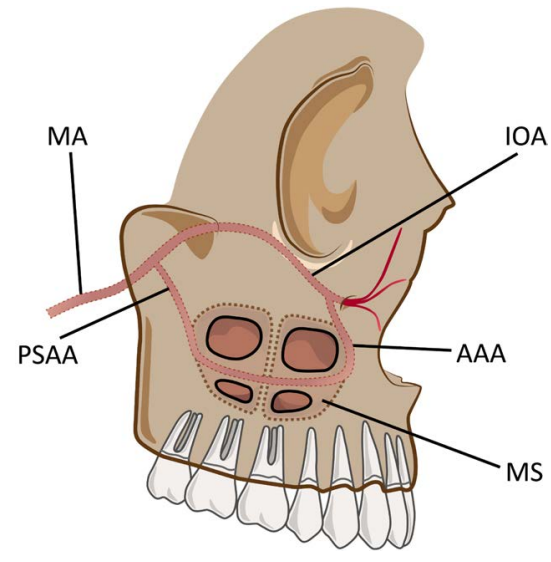

(b)

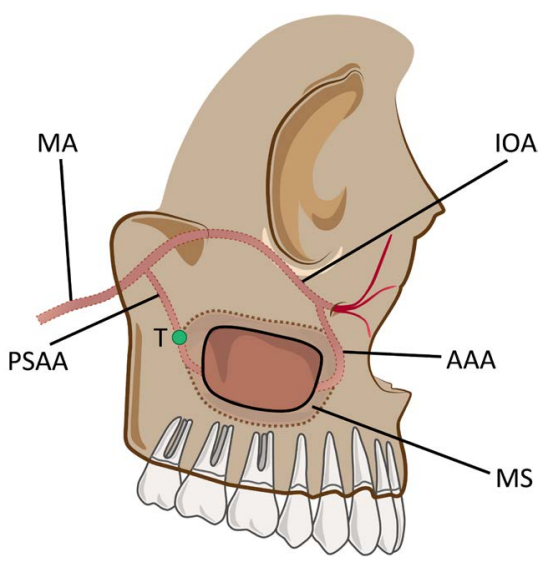

(c)

Figure 6. The double-window technique (a) traditionally used to avoid disruption of the alveolar antral artery. In the event of a septated sinus (b), this approach would then require a "quadruple-window", which would be difficult and not always feasible. Our proposed osteotomy protocol would use a bone scraper to intentionally lacerate the alveolar antral artery and tamponade a proximal site, T (c). AAA, alveolar antral artery; IOA, infraorbital artery; MA, maxillary artery; MS, maxillary sinus; PSAA, posterior superior alveolar artery.

\section{Discussion}

Regardless of whether vessel preservation or controlled vessel sacrifice is contemplated, all the above strategies share the same concerns:

1) They require management of this vessel at the same surgical site as the sinus lift itself. Inadvertent laceration is a potential outcome. Regardless of the original intended approach, once lacerated, salvage hemostatic measures and the risks they carry as discussed above, would then come into play.

2) When management is at the site of the osteotomy, it leaves a fragile fresh clot directly within the surgical site. Subsequent manipulation of the area can retrigger the bleed, leading to another round of bleeder management. Ultimately this can lead to surgery cancellation and rescheduling.

It is an axiom of surgery that when a bleeder or anticipated bleeder is difficult to manage locally, then one would consider its disruption proximally. The classic 
definitive management of a vessel is ligation at a proximal site. However, the proximal segment of the AAA arising from the PSAA is intrabony $100 \%$ of the time, [11] [22] [44] and thereby not amenable to vessel clips without dissection. The origin of the PSAA starts as a branch of the maxillary artery in the pterygomaxillary fissure. We propose a simple means to cause an embolus of the AAA proximal to the surgical site without advanced surgical instrumentation.

\section{Proposed Approach}

Our proposed method disrupts the AAA proximally, causing a natural thromboembolus to occur (Figure 6(c)). The lateral posterior sinus wall, i.e. in the area of the third molar, is exposed as part of the surgical flap. Autogenous bone is harvested here using a bone scraper to create a shallow, wide-based trough with intention to be admixed with allograft or xenograft material (Figure 7(a)). The AAA is only covered by a thin layer of bone in this area, usually $<1 \mathrm{~mm}$ in thickness. Its location can be predetermined on a CBCT. In cases with larger caliber vessels or thinner maxillary bone, the vessel path can be directly visualized (Figure 7(b)). This scraping will eventually rupture the vessel, creating a pinpoint pulsatile bleed, in a convenient location away from desired osteotomy site. Gauze can then be packed here to stop the brisk bleeding of the exposed AAA. As only a pinpoint area of vessel is exposed and the sinus membrane is still protected by an intact sinus wall, there is no risk of perforating the sinus membrane with this packing being done aggressively. This pack will then be retained under the retracted flap and ignored for the remainder of the procedure. The originally intended osteotomy site can now be approached and the now defunct artery previously impeding surgery can be ignored (Figure $7(\mathrm{c})$ ). At the end of the procedure, the vessel is hemostatic and requires no additional management. An overview of the workflow is presented (Figure 8).

\section{Potential Drawbacks}

Sacrificing the AAA raises concerns of ischemia to the graft site. There is no consensus in the literature on the importance of vessel preservation. Advocates for preservation feel it is needed to support bone graft neoangiogenesis but this approach requires significant additional time, expertise, and equipment [45]. It is our opinion that this is unnecessary because the head and neck region is very well vascularized with collaterals and arterial disruptions of minor branches are very well tolerated. While no statistical studies have examined the outcomes of AAA sacrifice to our knowledge, in the authors' experience, all cases with visible AAAs on CBCT for the past five years (20\% of our cases) have been sacrificed with no difference in graft and implant success rate between those with sacrificed AAAs vs. those without. There are no extraneous intra- or post-operative complications as bone scraping is an inherent step of sinus lifts. Besides this bone scraper, no additional costs or equipment beyond the standard orofacial surgical armamentarium is required. 


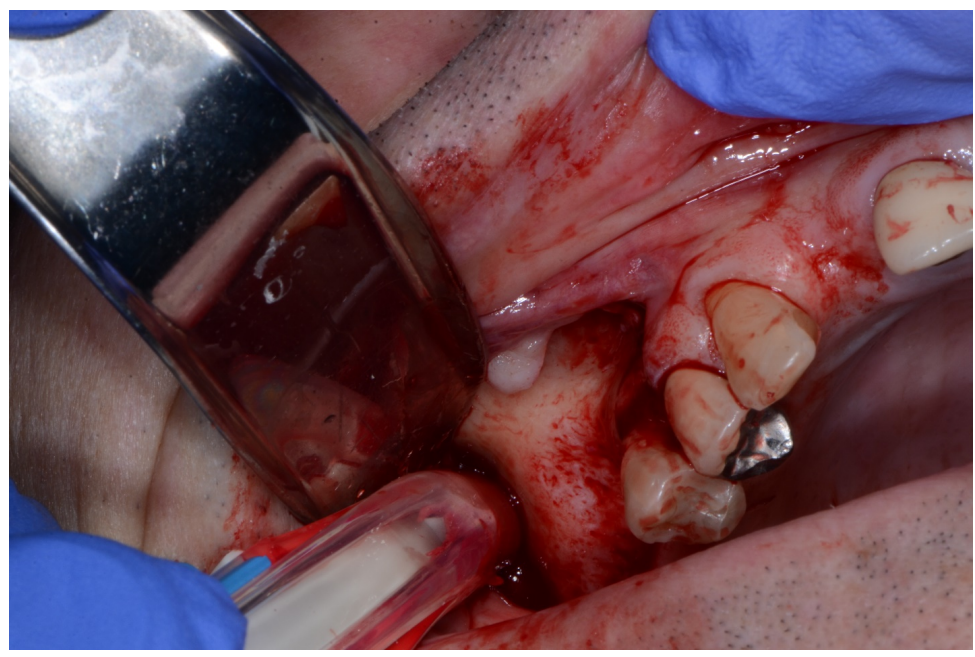

(a)

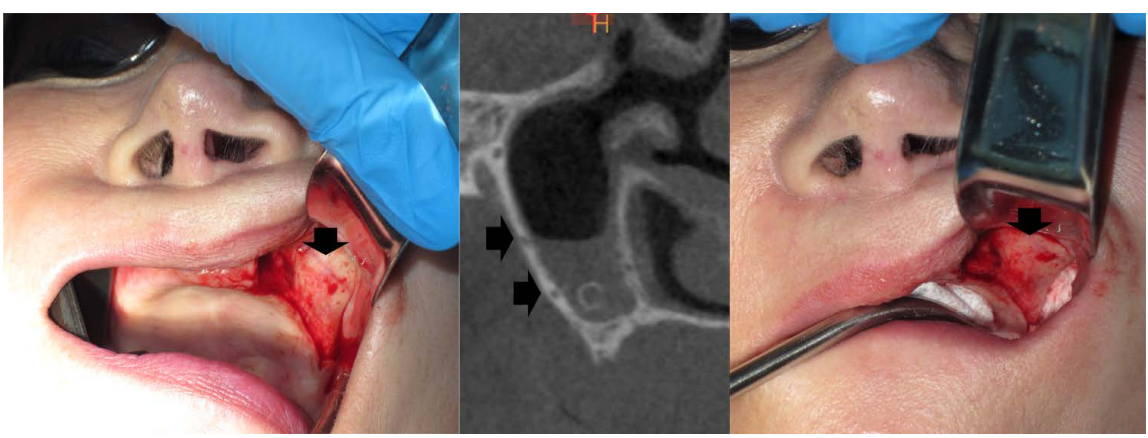

(b)

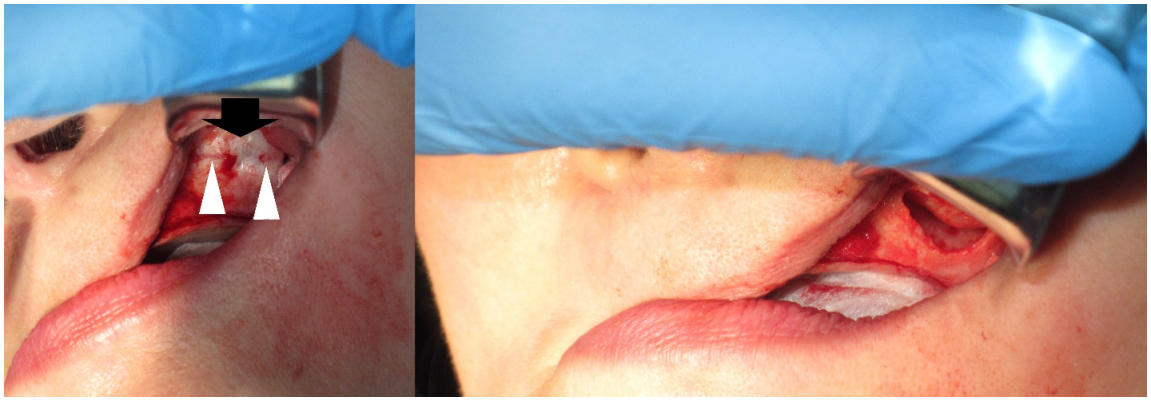

(c)

Figure 7. A bone scraper is used to harvest bone from the posterior maxilla for later use while simultaneously creating a controlled, pinpoint bleed of the alveolar antral artery (a). The intrabony path of the vessel is seen traversing the planned osteotomy site (b). On cone bean computed tomography, this patient had a bifid vessel (black arrows). The superior vessel is closer to the bony surface and is visible through the bone while the inferior vessel is not as appreciable clinically. Both would potentially be ruptured by a traditional approach. Our proposed osteotomy protocol (c) would neutralize the proximal source of the artery with gauze packing. The posterior portion of the vessel (posterior white arrowhead) is no longer visible in bone but the anterior portion (anterior white arrowhead) that has collateral flow from the infraorbital artery remains visible. During the osteotomy, the alveolar antral artery is intentionally lacerated, with limited resultant flow from the anterior portion and no bleeding from the posterior. The remaining procedure continues without further arterial bleeding despite the osteotomy being large enough to rupture both bifid vessel branches. 


\section{Proposed approach}

\section{Plan to sacrifice vessel Plan to preserve vessel}

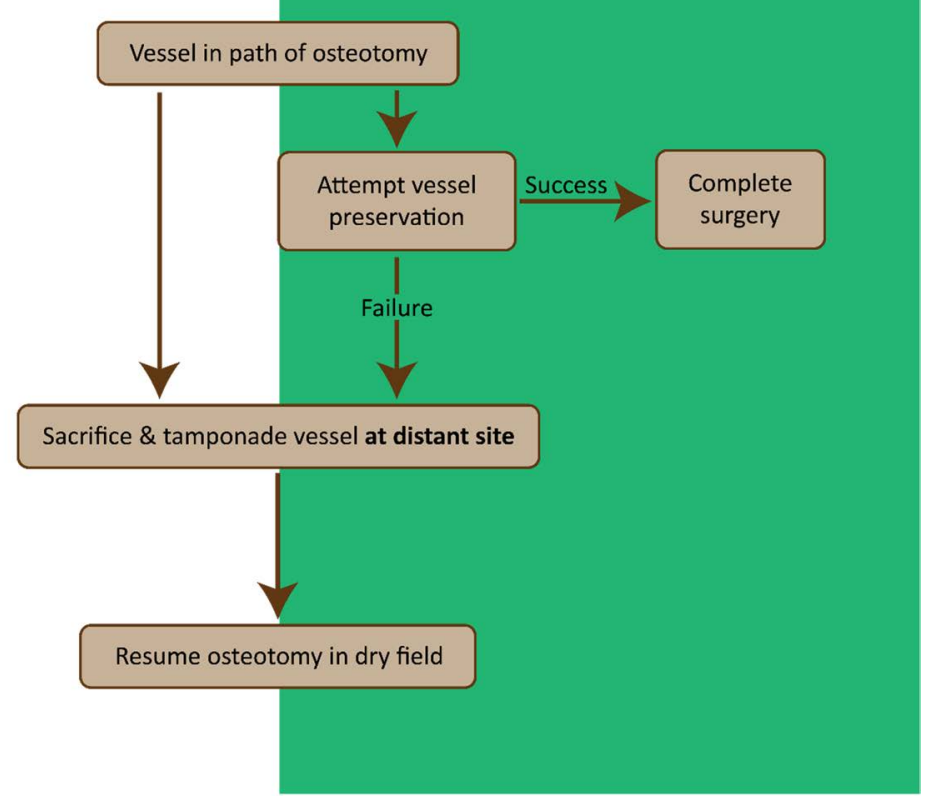

Figure 8. Workflow of the proposed osteotomy protocol. Preservation of the alveolar antral artery can be attempted in either case, but inadvertent laceration may occur if it is not preemptively sacrificed. Tamponade at a proximal site results in a dry surgical field by managing the vessel prophylactically or as a salvage procedure.

\section{Conclusion}

We have described a novel method for AAA management during sinus lift surgery. Compared to classic methods of managing this bleeder at the site of osteotomy, this proposed approach is likely faster, requires minimal to no additional equipment or expertise, allows surgery to go on uninterrupted while the tamponade is occurring at a distant site, and eliminates the need to manage an arterial bleed in the proximity of the sinus membrane. The risk of sinus membrane perforation is decreased. We hope it will simplify sinus lift surgery for our other surgical colleagues as it has for us. The AAA is an under-reported source of surgical complications and warrants further research.

\section{Conflicts of Interest}

The authors declare no conflicts of interest regarding the publication of this paper.

\section{References}

[1] Tatum, H. (1986) Maxillary and Sinus Implant Reconstructions. Dental Clinics of North America, 30, 207-229.

[2] Chipaila, N., Marini, R., Sfasciotti, G.L., Cielo, A., Bonanome, L. and Monaco, A. 
(2014) Graftless Sinus Augmentation Technique with Contextual Placement of Implants: A Case Report. Journal of Medical Case Reports, 8, 437. https://doi.org/10.1186/1752-1947-8-437

[3] Bedrossian, E., Rangert, B., Stumpel, L. and Indresano, T. (2006) Immediate Function with the Zygomatic Implant: A Graftless Solution for the Patient with Mild to Advanced Atrophy of the Maxilla. The International Journal of Oral \& Maxillofacial Implants, 21, 937-942.

[4] Varela-Centelles, P., Loira, M., González-Mosquera, A., et al. (2020) Study of Factors Influencing Preoperative Detection of Alveolar Antral Artery by CBCT in Sinus Floor Elevation. Scientific Reports, 10, Article No. 10820.

https://doi.org/10.1038/s41598-020-67644-9

[5] Chiapasco, M., Casentini, P. and Zaniboni, M. (2009) Bone Augmentation Procedures in Implant Dentistry. The International Journal of Oral \& Maxillofacial Implants, 24, 237-259.

[6] Maridati, P., Stoffella, E., Speroni, S., Cicciu, M. and Maiorana, C. (2014) Alveolar Antral Artery Isolation during Sinus Lift Procedure with the Double Window Technique. The Open Dentistry Journal, 8, 95-103.

https://doi.org/10.2174/1874210601408010095

[7] Lee, C.Y.S. (2010) Brisk, Prolonged Pulsatile Hemorrhage during the Sinus Graft Procedure: A Case Report with Discussion on Intra-Operative Hemostatic Management. Implant Dentistry, 19, 189-195.

https://doi.org/10.1097/ID.0b013e3181dec0c7

[8] Elian, N., Wallace, S., Cho, S.-C., Jalbout, Z.N. and Froum, S. (2005) Distribution of the Maxillary Artery as It Relates to Sinus Floor Augmentation. The International Journal of Oral \& Maxillofacial Implants, 20, 784-787.

[9] Rahpeyma, A. and Khajehahmadi, S. (2015) Open Sinus Lift Surgery and the Importance of Preoperative Cone-Beam Computed Tomography Scan: A Review. Journal of International Oral Health, 7, 127-133.

[10] Balaguer-Martí, J.-C., Peñarrocha-Oltra, D., Balaguer-Martínez, J. and Peñarrocha-Diago, M. (2015) Immediate Bleeding Complications in Dental Implants: A Systematic Review. Medicina Oral, Patologia Oral, Cirugia Bucal, 20, e231-e238. https://doi.org/10.4317/medoral.20203

[11] Rosano, G., Taschieri, S., Gaudy, J.-F., Weinstein, T. and Del Fabbro, M. (2011) Maxillary Sinus Vascular Anatomy and Its Relation to Sinus Lift Surgery. Clinical Oral Implants Research, 22, 711-715. https://doi.org/10.1111/j.1600-0501.2010.02045.x

[12] Rysz, M., Ciszek, B., Rogowska, M. and Krajewski, R. (2014) Arteries of the Anterior Wall of the Maxilla in Sinus Lift Surgery. International Journal of Oral and Maxillofacial Surgery, 43, 1127-1130. https://doi.org/10.1016/j.ijom.2014.02.018

[13] Ella, B., Sédarat, C., Noble, R.D.C., et al. (2008) Vascular Connections of the Lateral Wall of the Sinus: Surgical Effect in Sinus Augmentation. The International Journal of Oral \& Maxillofacial Implants, 23, 1047-1052.

[14] Chan, H.-L. and Wang, H.-L. (2011) Sinus Pathology and Anatomy in Relation to Complications in Lateral Window Sinus Augmentation. Implant Dentistry, 20, 406-412. https://doi.org/10.1097/ID.0b013e3182341f79

[15] Rahpeyma, A., Khajehahmadi, S. and Amini, P. (2014) Alveolar Antral Artery: Does Its Diameter Correlate with Maxillary Lateral Wall Thickness in Dentate Patients? Iranian Journal of Otorhinolaryngology, 26, 163-167.

[16] Kim, J.H., Ryu, J.S., Kim, K.-D., Hwang, S.H. and Moon, H.S. (2011) A Radio- 
graphic Study of the Posterior Superior Alveolar Artery. Implant Dentistry, 20, 306-310. https://doi.org/10.1097/ID.0b013e31822634bd

[17] Testori, T., Rosano, G., Taschieri, S. and Del Fabbro, M. (2010) Ligation of an Unusually Large Vessel during Maxillary Sinus Floor Augmentation. A Case Report. European Journal of Oral Implantology, 3, 255-258.

[18] Jensen, S.S., Eriksen, J. and Schiodt, M. (2012) Severe Bleeding after Sinus Floor Elevation Using the Transcrestal Technique: A Case Report. European Journal of Oral Implantology, 5, 287-291.

[19] Albuquerque, D., Manhães, J.L., Silva, M., Francischone, C., Franco, A. and Junqueira, J. (2020) Alveolar Antral Artery in Edentulous Patients and Their Visualization through Cone Beam Computed Tomography. Morphologie, 105, 64-68. https://doi.org/10.1016/j.morpho.2020.08.003

[20] Kolte, R.A., Kolte, A.P., Rahate, P.S. and Bawankar, P.V. (2020) Association of Location and Diameter of Alveolar Antral Artery to Crest of Alveolar Bone in Dentate and Partially Edentulous Patients-A Cone-Beam Computed Tomography Study. Journal of Indian Society of Periodontology, 25, 55-60. https://doi.org/10.4103/jisp.jisp_603_19

[21] Laovoravit, V., Kretapirom, K. and Pornprasertsuk-Damrongsri, S. (2020) Prevalence and Morphometric Analysis of the Alveolar Antral Artery in a Group of Thai Population by Cone Beam Computed Tomography. Oral Radiology. https://doi.org/10.1007/s11282-020-00478-3

[22] Solar, P., Geyerhofer, U., Traxler, H., Windisch, A., Ulm, C. and Watzek, G. (1999) Blood Supply to the Maxillary Sinus Relevant to Sinus Floor Elevation Procedures. Clinical Oral Implants Research, 10, 34-44. https://doi.org/10.1034/j.1600-0501.1999.100105.x

[23] Varela-Centelles, P., Loira-Gago, M., Gonzalez-Mosquera, A., Seoane-Romero, J.-M., Garcia-Martin, J.-M. and Seoane, J. (2016) Distance of the Alveolar Antral Artery from the Alveolar Crest. Related Factors and Surgical Considerations in Sinus Floor Elevation. Medicina Oral, Patologia Oral, Cirugia Bucal, 21, e758-e765. https://doi.org/10.4317/medoral.21475

[24] Park, W.-H., Choi, S.-Y. and Kim, C.-S. (2012) Study on the Position of the Posterior Superior Alveolar Artery in Relation to the Performance of the Maxillary Sinus Bone Graft Procedure in a Korean Population. Journal of the Korean Association of Oral and Maxillofacial Surgeons, 38, 71.

https://doi.org/10.5125/jkaoms.2012.38.2.71

[25] Varela-Centelles, P., Seoane, J., Loira-Gago, M., González-Mosquera, A. and Seoane-Romero, J.M. (2017) Diameter of Alveolar Antral Artery in the Lateral Sinus Wall: Study of Related Factors. British Journal of Oral and Maxillofacial Surgery, 55, 413-415. https://doi.org/10.1016/j.bjoms.2016.12.001

[26] Rodella, L.F., Labanca, M., Boninsegna, R., Favero, G., Tschabitscher, M. and Rezzani, R. (2010) Intraosseous Anastomosis in the Maxillary Sinus. Minerva Stomatologica, 59, 349-354.

[27] Valente, N.A. (2016) Anatomical Considerations on the Alveolar Antral Artery as Related to the Sinus Augmentation Surgical Procedure. Clinical Implant Dentistry and Related Research, 18, 1042-1050. https://doi.org/10.1111/cid.12355

[28] Kang, S.-J., Shin, S.-I., Herr, Y., Kwon, Y.-H., Kim, G.-T. and Chung, J.-H. (2013) Anatomical Structures in the Maxillary Sinus Related to Lateral Sinus Elevation: A Cone Beam Computed Tomographic Analysis. Clinical Oral Implants Research, 24, 75-81. https://doi.org/10.1111/j.1600-0501.2011.02378.x 
[29] Mardinger, O., Abba, M., Hirshberg, A. and Schwartz-Arad, D. (2007) Prevalence, Diameter and Course of the Maxillary Intraosseous Vascular Canal with Relation to Sinus Augmentation Procedure: A Radiographic Study. International Journal of Oral and Maxillofacial Surgery, 36, 735-738. https://doi.org/10.1016/j.ijom.2007.05.005

[30] Ilgüy, D., Ilgüy, M., Dolekoglu, S. and Fisekcioglu, E. (2013) Evaluation of the Posterior Superior Alveolar Artery and the Maxillary Sinus with CBCT. Brazilian Oral Research, 27, 431-437. https://doi.org/10.1590/S1806-83242013000500007

[31] Bernardi, S., Mummolo, S., Ciavarelli, L.M., Li Vigni, M., Continenza, M.A. and Marzo, G. (2016) Cone Beam Computed Tomography Investigation of the Antral Artery Anastomosis in a Population of Central Italy. Folia Morphologica (Warsz), 75, 149-153. https://doi.org/10.5603/FM.a2015.0095

[32] Kalabalik, F. and Akçay, H. (2019) Evaluation of the Alveolar Antral Artery Position in the Lateral Sinus Wall Using Cone-Beam Computed Tomography. Annals of Clinical and Analytical Medicine, 11, 330-334. https://doi.org/10.4328/ACAM.20084

[33] Ferneini, E.M. and Bennett, J.D. (2018) Perioperative Assessment of the Maxillofacial Surgery Patient: Problem-Based Patient Management. Springer International Publishing, Berlin. https://www.springer.com/gp/book/9783319588674 https://doi.org/10.1007/978-3-319-58868-1

[34] Flanagan, D. (2005) Arterial Supply of Maxillary Sinus and Potential for Bleeding Complication during Lateral Approach Sinus Elevation. Implant Dentistry, 14, 336-338. https://doi.org/10.1097/01.id.0000188437.66363.7c

[35] Onlineredaktion QV-G Berlin, Testori/del Fabbro/Weinstein, et al. (2019) Maxillary Sinus Surgery and Alternatives in Treatment. Quintessence Publishing, Berlin. https://www.quintessenz.de/books.php?idp=14431\&qtw_language=en

[36] Jung, J., Yim, J.-H., Kwon, Y.-D., et al. (2011) A Radiographic Study of the Position and Prevalence of the Maxillary Arterial Endosseous Anastomosis Using Cone Beam Computed Tomography. The International Journal of Oral \& Maxillofacial Implants, 26, 1273-1278.

[37] Valente, N.A., Harogiannis, K. and Andreana, S. (2015) Alveolar Antral Artery Management during Sinus Elevation: A Case Report of a Novel Approach with Review of the Literature. International Journal of Contemporary Dental and Medical Reviews, 2015, Article ID: 100215.

[38] Rahpeyma, A. and Khajehahmadi, S. (2018) Indications for Palatal Sinus Lift: Case Series. Journal of Indian Society of Periodontology, 22, 254-256.

https://doi.org/10.4103/jisp.jisp_357_17

[39] Maridati, P., Stoffella, E., Speroni, S., Cicciu, M. and Maiorana, C. (2014) Alveolar Antral Artery Isolation during Sinus Lift Procedure with the Double Window Technique. The Open Dentistry Journal, 8, 95-103.

https://doi.org/10.2174/1874210601408010095

[40] Orhan, K., Kusakci, S.B., Aksoy, S., Bayindir, H., Berberoğlu, A. and Seker, E. (2013) Cone Beam CT Evaluation of Maxillary Sinus Septa Prevalence, Height, Location and Morphology in Children and an Adult Population. Medical Principles and Practice, 22, 47-53. https://doi.org/10.1159/000339849

[41] Neugebauer, J., Ritter, L., Mischkowski, R.A., et al. (2010) Evaluation of Maxillary Sinus Anatomy by Cone-Beam CT Prior to Sinus Floor Elevation. The International Journal of Oral \& Maxillofacial Implants, 25, 258-265.

[42] Lana, J.P., Carneiro, P.M.R., Machado V de, C., de Souza, P.E.A., Manzi, F.R. and 
Horta, M.C.R. (2012) Anatomic Variations and Lesions of the Maxillary Sinus Detected in Cone Beam Computed Tomography for Dental Implants. Clinical Oral Implants Research, 23, 1398-1403. https://doi.org/10.1111/j.1600-0501.2011.02321.x

[43] Shahidi, S., Zamiri, B., Momeni, D.S., Salehi, S. and Hamedani, S. (2016) Evaluation of Anatomic Variations in Maxillary Sinus with the Aid of Cone Beam Computed Tomography $(\mathrm{CBCT})$ in a Population in South of Iran. Journal of Dentistry (Shiraz), 17, 7-15.

[44] Traxler, H., Windisch, A., Geyerhofer, U., Surd, R., Solar, P. and Firbas, W. (1999) Arterial Blood Supply of the Maxillary Sinus. Clinical Anatomy, 12, 417-421. https://doi.org/10.1002/(SICI)1098-2353(1999)12:6<417::AID-CA3>3.0.CO;2-W

[45] Taschieri, S. and Rosano, G. (2010) Management of the Alveolar Antral Artery during Sinus Floor Augmentation Procedures. Journal of Oral and Maxillofacial Surgery, 68, 230. https://doi.org/10.1016/j.joms.2009.06.036 